\title{
Position-based Selective Neighbors
}

\author{
Sofian Hamad ${ }^{1}$, Taoufik Yeferny ${ }^{2}$, Salem Belhaj ${ }^{3}$ \\ Computer Science Department, Northern Border University \\ Arar Kingdom of Saudi Arabia
}

\begin{abstract}
In this paper, we propose a routing protocol, named Position-based Selective Neighbors (PSN), for controlling the Route Request (RREQ) propagation in Mobile Ad-hoc Networks (MANETs). PSN relies on the Residual Energy (RE) and the Link Lifetimes (LLT) factors to select the better end-toend paths between mobile nodes. The key concept is to consider the RE and the LLT to find the best neighboring nodes to forward the received RREQs. A Simulation has been performed to compare PSN with other pioneering routing protocols. Experimental results show that PSN performs better than its competitors. Indeed, our protocol increases the network life time and reduces the network overhead. Furthermore, it reduces the overhead generated by the redundant RREQ, while maintaining good reachability among the mobile nodes.
\end{abstract}

Keywords-Mobile Ad-hoc network (MANET); routing protocol; energy aware; link life time; $A O D V$

\section{INTRODUCTION}

Academics and industry have become increasingly interested in wireless research over the last decade. Wireless access was chosen because it allows free movement. A Mobile Ad-hoc Network (MANET) has proved very interesting in finding ways to improve its operation and performance. A MANET typically consists of interconnected mobile nodes using wireless links that have no access points or permanent infrastructure. Moreover, a lot of work has been performed across the layers of Open Systems Interconnection (OSI) while applying Medium Access Control (MAC). Particularly, many routing algorithms have been proposed to provide endto-end routes. These can be reliable and robust against the mobility of the nodes.

For example, neighboring nodes in wireless networks share wireless media. In addition to this, the nodes must compete with others in order to gain access to these media (channels). A MAC layer will control such an operation. Basically, the MAC protocol governs the access of wireless devices to shared wireless media. This protocol imposes many time constraints in the process to properly regulate the shared resource and to avoid collisions. These can happen, as illustrated in Figure 1 In such a case, the node A does not know that the node $\mathrm{B}$ simultaneously receives data from the node C. As a result, it can start its own transmission, which will cause the collision with node $\mathrm{B}$. The neighboring-node collision and interference, the hidden-node presence as well as the distances between senders and receivers have a significant effect on wireless network performances. MANETs face such a problem, particularly while having a lot of data, controlling packet traffic as well as mobile topology.

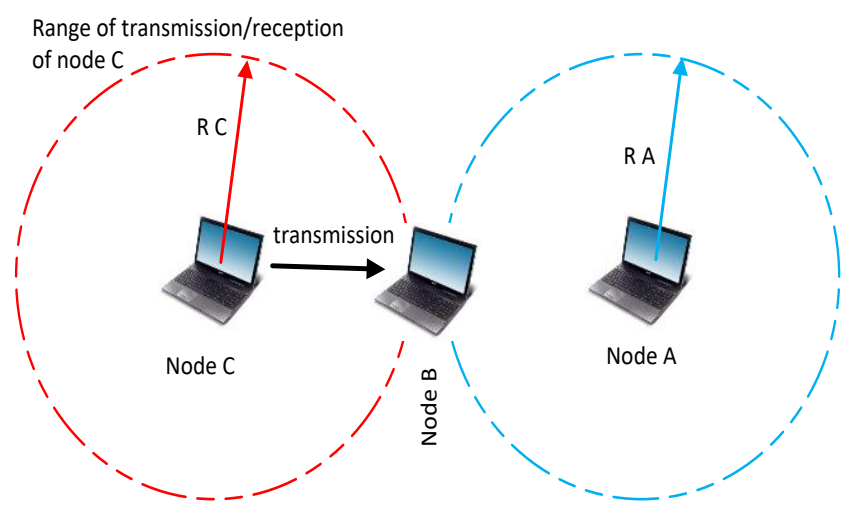

Fig. 1. Instance of Hidden Node Problem.

Because MANET's topology is highly mobile and the nodes generating data and forwarding entities within networks, designing efficient and robust routing protocols requires a lot of effort. Several routing protocols have been recently put forward for MANETs, whose goal is to establish end-to-end paths in multi-hop scenarios between sink destination nodes and data-generating sources [1-6]. Nodes discover routes to a specific destination within conventional on-demand routing protocols [4-5], through the broadcasting of a Route Request (RREQ) packet. On the reception of a RREQ, the node will check if that packet was previously received. If it is the case, the node will drop it. If it is the contrary, a Route Reply (RREP) will be then sent back to the source node according to the availability of the route. In either case, the RREQ will be rebroadcasted by this node to its immediate neighbors until finding its destination. As a matter of fact, that route-discovery method is called blind flooding. The rebroadcasting of a copy of the received RREQ by each mobile node results, within the global network, in a maximal $\mathrm{N}-2$ number of rebroadcasts. In such a situation, $\mathrm{N}$ in this network is the number of nodes. Thus, there is possibly excessive redundant retransmission, hence high channel contention. This may lead to excessive packet collisions within dense networks. This can be called a broadcast storm problem [7], so it greatly raises end-to-end delay and network communication overhead, whilst rising bandwidth loss [7,9].

A lot of existing approaches have attempted to resolve the flooding problem through the reduction in the number of redundant messages. On the other hand, this results in low coverage and connectivity degree. In fact, the interdependence between both phenomena is problematic for balancing message overhead (in other words, that redundancy level) and coverage [8]. 
Therefore, the decrease in collisions within the network is able to ameliorate network performances, mainly for MANETs, where nodes may collaborate together so as to connect to those nodes not actually being within their transmission range. In addition to that, broadcasting RREQ messages generate duplicate messages across the full network, looking at the same time for an end-to-end path, hence a big chance of potential collisions. The elimination of the unnecessary RREQ packets is able to decrease the number of packet collisions, which will ameliorate the network performance.

This paper puts forward one novel algorithm which minimizes within the global network the RREQ propagation and simultaneously keeps the network connectivity. The $(\mathrm{x}, \mathrm{y})$ coordinates of all nodes and their neighbors in the suggested algorithm are known. According to those positions, the best neighbors are selected by one node to further rebroadcast RREQs. We divide the source node's transmission range to 4 equal zones. The latter are as follows: (Zone1, Zone2, Zone3 and Zone4) in a set $M=\{M 1, M 2, M 3, M 4\}$. Furthermore, we select 4 neighbor nodes from these zones based on the quality of their links to the source node as well as on their residual energy levels.

Our workout lines one effective routing protocol, which can tackle such a flooding problem and minimize RREQs propagation when at the same time maintaining among nodes within a global network comparable reachability.

\section{RELATED WORK}

Several approaches have recently proposed manners of decreasing the broadcast-storm effect due to simple flooding [7-10]. Indeed, we can classify these approaches to five categories [8]: the neighbor knowledge methods, the probability-based approach, the position-based methods, simple flooding and different other approaches utilizing various techniques. The simple flooding has been discussed earlier in the introduction

\section{A. Neighbor Knowledge Methods}

From a first point of view, a main concept for this method is expanding data concerning the node neighbors. According to that, every node sends a neighbor node address or two ones to their neighbors. After that, this node uses existing "hello" messages in the purpose of sending this information periodically. As a result, every node can implicitly know what is in common with others. In the same vein, the writers of [11] suggested the two-hop backward-neighbor information concept. This latter was utilized to minimize the number of forwarding nodes. It also reduced the collisions in the network. Generally, such a suggested mechanism required exchanging one-hop hello messages. A novel joint one-hop neighbor information-based flooding scheme was put forward in [12], consisting of two sub-algorithms: receiver-phase and sender-phase. The sender-phase algorithm would facilitate for the node the selection of one subset of its one-hop neighbors in order to forward flooding messages. It would also select forwarding nodes that could greatly contribute to flooding message dissemination. The writers in [13] put forward an efficient flooding scheme. Indeed, this latter was based on one-hop Information within MANETs. Basically, every node would use its one-hop neighbor data. Looking for one new route, every node would determine a subset of its neighbors as candidate ones for rebroadcasting that message when they received it. Accordingly, the addresses of these nodes were attached to the RREQ message. Once a RREQ was received, the node would search for its address. When the latter was found by the receiving node, the sending one would provide the candidates from a novel subset of its neighbors and would rebroadcast the RREQ. If it was not the case, the node would drop the RREQ.

From a second point of view, neighbor-knowledge methods succeed in the reduction of extra RREQs in the network. On the other hand, the addresses of all the neighboring nodes are carried by periodic hello messages, hence the use of the available bandwidth, which might rise up the overhead. In addition, because of the nodes mobility, the gathered two-hop or one-hop data are not all the time exact.

\section{B. Position-based Methods}

It is worth mentioning that area-based methods comprise location-based and distance-based schemes. These methods show the area that can be offered by one node when rebroadcasting the same received message. In fact, in the transmission range of that node, a great distance from a previous broadcasting node will result in an additional coverage to be acquired, hence a big chance to reach more nodes. Actually, the writers put forward in [14] an approach which is known as Flooding based on One-hop Neighbor Information and Adaptive Holding (FONIAH).

The authors could assume that nodes knew their geographical location. Added to that, the sharing positions among the nodes would require that every node sent hello messages continuously having location information. One main idea of FONIAH is the node's ability to select those furthest nodes within its transmission range. Afterwards, it could calculate the distance (Maximum Distance (Dmax)) between these furthest nodes and itself. Such a distance was utilized for calculating waiting time at that receiver node. Abolhasan and Wysocki suggested in [15] Position-based Selective Flooding (PSF), where one novel scheme was applied to select forwarding nodes. Mainly, a received RREQ Would be rebroadcast by the receiving nodes just as it enters the Forwarding Region (FR), as it is illustrated in Fig. 2. That was a good position from which neighbors could rebroadcast RREQs, and therefore there would be a strong signal will probably be and a great coverage area. However, such a technique might not find the requested destination for the reason that the destination node was opposing the forwarder.

Hamad et al. suggested in $[16,27]$ a new algorithm for the reduction of overhead generated by redundant RREQ messages. Part of their work, candidate Neighbors rebroadcasting a RREQ (CNRR) would divide the transmission ranges. The latter were done for nodes sending or rebroadcasting those RREQs into four equal zones (Zone1, Zone2, Zone3 and Zone4). So after that, a node per zone would be selected in the aim of rebroadcasting the RREQ. This selection was on the basis of distance between a node and its neighbors. 


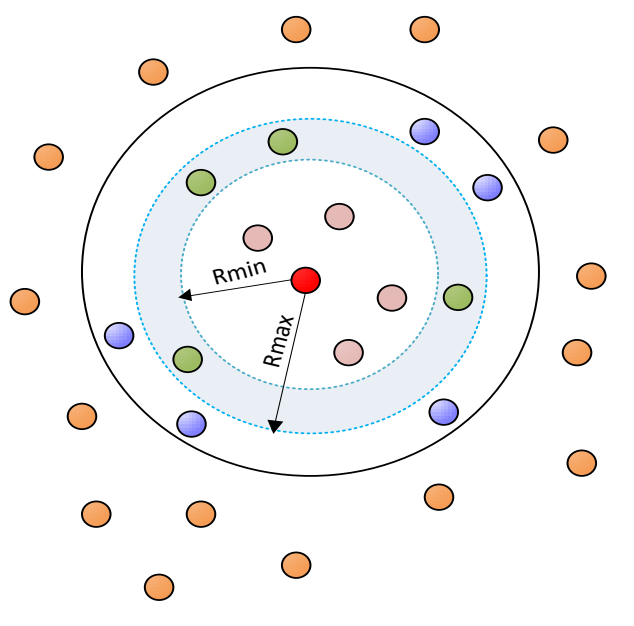

The Forwarder nodes as falling in Forwarding Region

Fig. 2. Illustration of FR in PSF Method.

\section{Probability-based Approaches}

They are contingent with assigning various nodeparticipation probabilities within a network. These probabilities are signs to nodes for discarding or rebroadcasting a received RREQ. Their values are able to differ for multiple algorithms and node conditions. Yassein et al. suggested in [17] a new probabilistic flooding algorithm that can build up the threshold value for a node having many neighbors. As a consequence, this node cannot rebroadcast the received RREQ. On the other hand, this node may rebroadcast the received RREQ in condition of having a low number of neighbor nodes. Nourazar et al. proposed in [18] a Dynamic Adjusted Probabilistic Flooding (DAPF) Algorithm. Its main goal was to rebroadcast the probability function one message dynamically adjustable with local observations and passing time. We can cite for instance the number of received duplicate messages and network density. Kim et al. suggested in [19] one dynamic probabilistic broadcasting approach. This latter was composed of two (probabilistic and position-based) methods. The probability here was assigned to nodes upon the basis of their distances from a RREQ sender. As a result, in case the receiver node was near that sender node, it might be difficult to rebroadcast the RREQ. Otherwise, it would be more probable to rebroadcast the RREQ and to achieve a wider coverage area.

\section{Other Approaches}

As it seems to be, various other approaches have been also considered by the research community in the objective of tackling the broadcast storm problem. For example, both studies of [20-21] considered node speed necessary to rebroadcast the RREQs.

Khamayseh et al. suggested in [20] two approaches for the enhancement of the route discovery phase and for the increase in overall routing performance. In addition, the authors considered node speed necessary to participate at the route discovery phase. Both approaches were Aggregate-AODV (Agg-AODV) and Per-Hop Mobility Aware (PH-MAAODV), where the node would keep track of its speed.

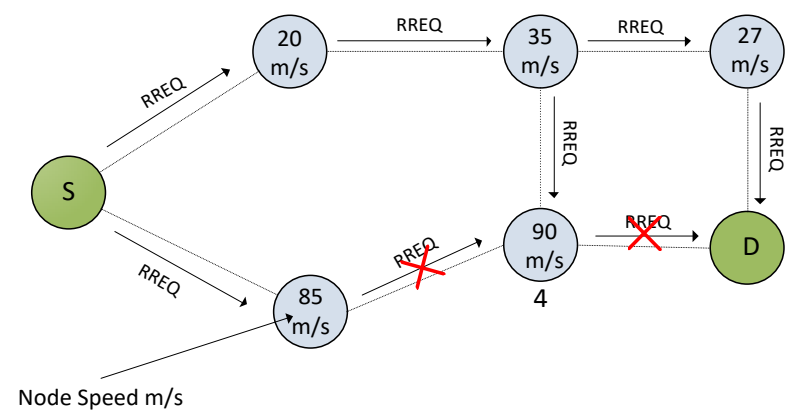

Connection between the Nodes

High Mobile Node (The Node decided not to rebroadcast the RREQ)

Fig. 3. RREQ Propagation in PH-MA-AODV Method.

Firstly, in case a RREQ is received, the node decides if it will forward that RREQ based on its speed. So if the latter is high, the received RREQ will be discarded. In case it is low, the node will take the decision of participating in the route and forwarding, in a way or another, the received RREQ. These nodes are illustrated in Fig. 3. Their speed is greater than 80 $\mathrm{m} / \mathrm{s}$ and as a result, those received RREQs will be discarded. Secondly, the node attaches its speed. Afterwards, it will forward the received RREQ. Actually, the selection of the best route into the source node will be done by the destination node on the basis of the nodes' low aggregate speed.

\section{PROPOSED PSN PROTOCOL}

We will discuss in this part the PSN routing protocol. In view of fact, AODV as an on-demand routing protocol follows blind flooding in the purpose of disseminating route discovery packets in global networks. This blind flooding can work well when reachability very significant. Nevertheless, since end-toend route selection is carried out by these protocols utilizing hop counts, non-stable paths are able to return because of the MANET extremely mobile environment. To deal with such a problem, two solutions were proposed in [27]. The authors outlined a mechanism of placing into different zones sending/forwarding-node neighbors. The writers in [28] considered link stability and looked in an explicit manner into neighboring nodes' residual battery energy and quality of links. Such suggested protocols would decrease to a minimum the network-wide RREQ dissemination and at the same time preserve the desired connectivity. On the other hand, the previously proposed mechanisms had isolation problems. Let us take as an example the CNRR protocol. This latter just considered the locations of the neighboring nodes. In that way, the RREQ forwarding decisions were solely based upon distance. Despite the fact that RREQ dissemination considerably was reduced by that method, the energies of the remaining nodes and link quality were ignored. Consequently, these returned routes might not be stable for long. In the same context, during the route discovery phase, Link Stability and Energy Aware LSEA [28] protocols take into account nodes' residual energies and link quality. In fact, such a method returns stable paths, thus leading to a high throughput and fewer delays. Yet, because such a method does not give careful consideration to the positions of nodes when disseminating RREQs, we can compromise connectivity. 


\section{A. PSN Route Discovery Mechanism}

In the following, there are three phases of the route discovery process of the proposed PSN protocol:

First of all, the neighbors of the ' $\mathrm{S}$ ' node are divided ito 4 zones, so as to send an RREQ, which is in a precise manner the same mechanism in [27]. In addition, every neighbor $(\mathrm{x}, \mathrm{y})$ coordinates are made known for nodes by using specialized positioning devices like GPS [22].

Secondly, every one of the ' $S$ ' nodes will compare in a specific zone its average Link Lifetime (LLT) with the averaged LLT (LLTavg). This latter is got from all nodes' specific times. These nodes share the links with the current ' $S$ ' node. In a similar way, the ' $S$ ' node will compare in the specific zone all its neighbors' residual energies with (REavg).

Thirdly, a Candidate Node $(\mathrm{CN})$ will be selected by the ' $\mathrm{S}$ ' node among neighbors, based on specific conditions, in a specific zone in order to forward a current RREQ. This selection will be based on two conditions. Firstly, in condition that the neighboring nodes' LLTs and REs are higher than LLTavg and REavg, in that case this current node will be selected as a Potential Candidate Neighbor (PCN) and added to Potential Candidate List (PCL)of the ' $\mathrm{S}$ ' node. This is similar for PCL. Secondly, the ' $\mathrm{S}$ ' node will select $\mathrm{CNs}$ from an already existing PCL on the basis of their LLTs and REs. As a matter of fact, a node will be selected from a PCL set when having a great number of LLTs and Res, compared to other PCL nodes. On the other hand, if in the specific zone there is one ' $\mathrm{S}$ ' node neighboring node having the ability of meeting LLTavg and REavg conditions for PCLs, then such a node having in the specific zone most LLTs and REs will be selected as a $\mathrm{CN}$. This will be similar for all the zones.

As an example, let consider the MANET topology, as it is depicted in Fig. 4, where node $\mathrm{X}$ has the intention of sending a RREQ to its neighbors. Firstly, that node will divide its transmission range to four zones. It can be assumed that node X compares all its neighbors' LLTs and REs with LLTavg and REavg in Zone1. On the basis of the checks, nodes A, B and C will be in fact selected as Potential Candidate Neighbors, hence putting them within the PCL list. As a consequence, that node X PCL in Zone $1=\{\mathrm{A}, \mathrm{B}$ and $\mathrm{C}\}$. After that, the same node will select the best node in order to forward the RREQ while comparing LLTs and REs. Added to that, consider $($ LLTC and REC) > (LLTB and REB) > (LLTA and REA). In that case, that node $\mathrm{X}$ will select node $\mathrm{C}$, which will be considered as its $\mathrm{CN}$ in Zone1. In the same way, $\mathrm{CNs}$ will be selected within the other three zones. This will be done following the previously discussed procedure. Moreover, node $\mathrm{X}$ may have the capability of attaching all selected CNs addresses within RREQ packet respective zones. To make it clear, all nodes $\mathrm{X}$ zone neighbors will check whether their addresses are part of the address list upon receiving the RREQ packet. When they make sure their addresses are in the list, they can in fact forward the RREQ to their neighbors, This is done according to the PNS procedure. If it is not the case, the others will simply drop it.

In the objective of understanding the PSN route discovery mechanism concept, let us take into account Fig. 4 and Fig. 5, which, for simplicity, present just Zone1. In addition to that, The ' $\mathrm{S}$ ' node interactions with all its neighbors can be seen within the specific zone. As a matter of fact, $L_{s-A}, L_{s-B}, L_{s-D}, L_{s-E}$ and $L_{s-G}$ are respectively the neighboring (A, B, C, D, E and $G$ ) nodes links with the $S$ ' node. It should be noted that every node LLT is shown above the link, On the other hand, REs are below each individual node. As a result, every node knows its neighbors LLTs and REs.

It was suggested by the authors of [28, 29] every node knew all its neighbor nodes LLTs and REs by exchanging 'hello' messages. In a similar way, the 'hello' message, in the proposed PSN protocol, was modified to convey to all the current node neighbors its $(\mathrm{x}, \mathrm{y})$ coordinates and REs. Indeed, such a frequent exchange of 'hello' messages would certainly help every node to get new data concerning its neighbor's residual energy and link quality.

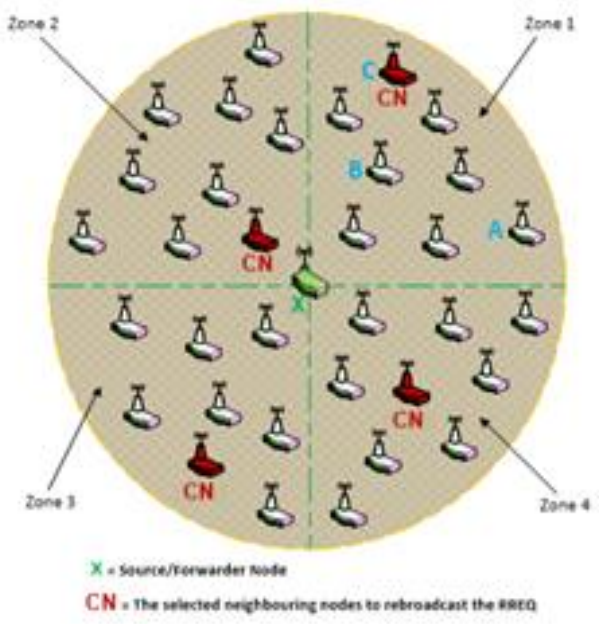

Fig. 4. Instance of Dividing Transmission Range into four Zones and Selecting CNs in Each Zone.

Fig. 5 shows the ' $\mathrm{S}$ ' node has the intention of sending a RREQ packet to its neighbors. According to that, after computing (neighboring nodes) LLTavg as well as REavg, there will be a comparison of these values with the LLT and RE values of every by the ' $S$ ' node in the target of discovering which nodes have LLTs and REs higher than that of LLTavg and REavg. In particular, just the $\mathrm{A}, \mathrm{E}$ and $\mathrm{F}$ nodes are included within the PCL.

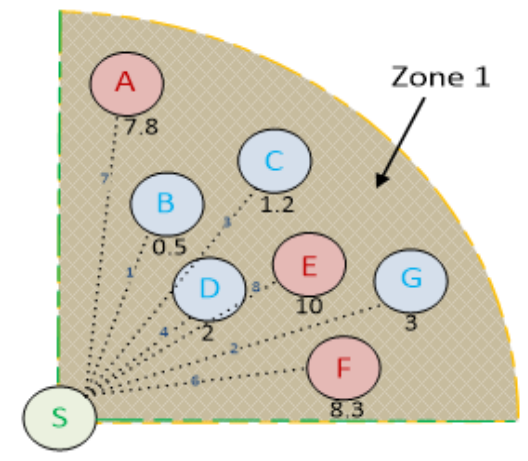

Fig. 5. Instance of Selecting best CN from PCL List in Specific Zone. 
From another point of view, the B, C, D and G nodes in Zone1will be left off the PCL due to the fact that LLTavg and REavg are higher than their LLTs and / or REs. Added to that, Fig.5 indicates that node E, among the PCNs within the PCL, is the best candidate to be selected like a $\mathrm{CN}$, which the case for node E, based on its good LLT and RE. That will be repeated by the ' $\mathrm{S}$ ' node for all the zones in a way that one node is selected in every zone like a $\mathrm{CN}$.

Finally, as a last phase, the ' $\mathrm{S}$ ' node will include all $\mathrm{CN}$ addresses and broadcast them. A similar RREQ will be received by all the zones nodes. Every time that these addresses are seen within an address list, the current RREQ will be rebroadcasted according to the aforementioned method. The other neighboring nodes will only drop that RREQ.

Table 1 shows that Algorithm 1 selects four $\mathrm{CNs}$ in the aim of forwarding the RREQ as follows. First of all, one full area around the ' $S$ ' node will be split up into four separate zones. The latter are symbolized by the $\mathrm{M}=\{\mathrm{M} 1, \mathrm{M} 2, \mathrm{M} 3$, M4\} set. As a matter of fact, a set of nodes inside every zone is represented by each member of set M. That is to say, M1 = $\left\{n_{1-\mathrm{M} 1} \quad, \quad n_{2-\mathrm{M} 1}, \ldots, n_{|\mathrm{M} 1|-\mathrm{M} 1}\right\} \quad, \quad \mathrm{M} 2=\left\{n_{1-\mathrm{M} 2}\right.$, $\left.n_{2-\mathrm{M} 2}, \ldots ., n_{|\mathrm{M} 2|-\mathrm{M} 2}\right\}, \mathrm{M} 3=\left\{n_{1-\mathrm{M} 3}, n_{2-\mathrm{M} 3}, \ldots ., n_{|\mathrm{M} 3|-\mathrm{M} 3}\right\}$, $\mathrm{M} 4=\left\{n_{1-\mathrm{M} 4}, n_{2-\mathrm{M} 4}, \ldots ., n_{|\mathrm{M} 4|-\mathrm{M} 4}\right\}$. Next, it iterates through every node of the specific zone and selects the PCL set and therefore the $\mathrm{CN}$ in that zone. In the end, the ' $\mathrm{S}$ ' node sends to chosen candidate nodes the RREQ packet.

\section{TABLE I. PSN ALGORITHM}

\begin{tabular}{|c|}
\hline 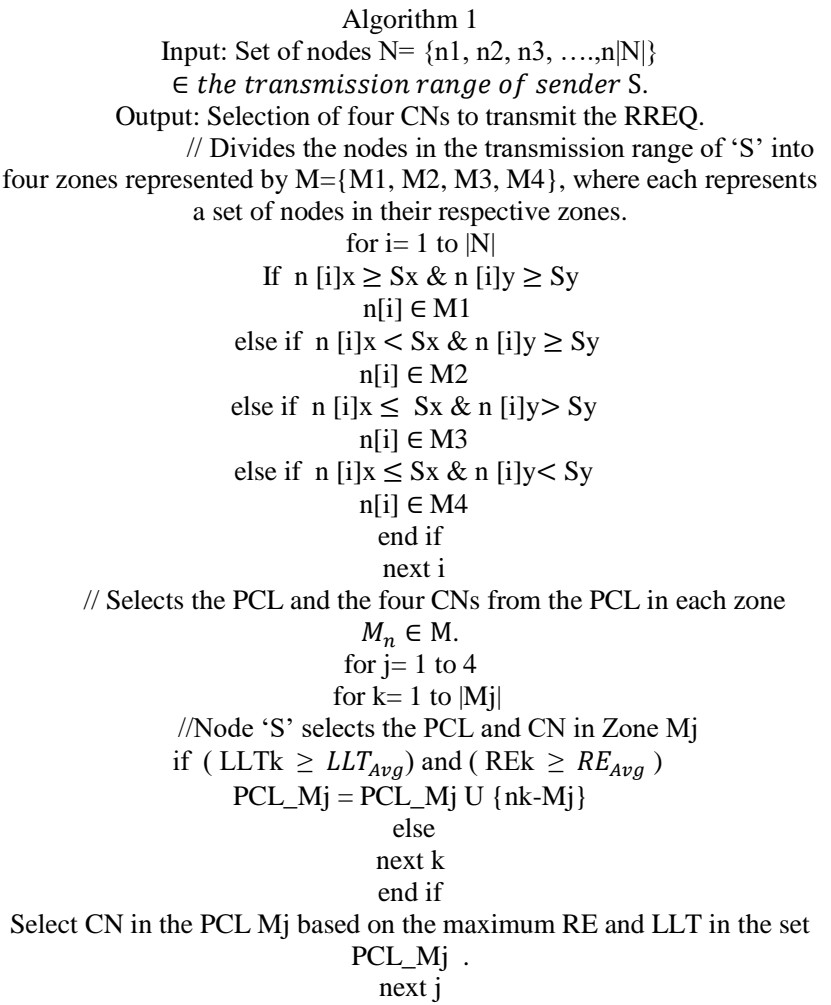 \\
\hline
\end{tabular}

\section{B. Percentage of RREQ Reception by Neighbour Nodes}

According to what has been discussed in [7], which we can get a $61 \%$ higher coverage area across a full network offered by rebroadcasting RREQs [7]. PSN will offer more betterment and enhancement with an algorithm which will also help CNs check for optimized RREQ dissemination. As an example, if Algorithm 1 is chosen to be run by any sender/forwarder ' $\mathrm{S}$ ' node, as represented and provided by Table 1 , four CNs will be then selected among its neighbors. In addition, the ' $\mathrm{S}$ ' node will attach the addresses of the selected CN to the RREQ packet and after that will broadcast it. Only the attached CNs will be permitted for further processing the received RREQ. This will happen if they find their addresses in a RREQ altered.

The verification of distances between every RREQ neighbor and the ' $\mathrm{S}$ ' sender will result in checking how many of their neighbors got a similar one. If the transmission range of ' $\mathrm{S}$ ' is more than the distance, the $\mathrm{CN}$ will assume that the neighbor obtained a similar RREQ as itself. Thus, any $\mathrm{CN}$ will be able to get the percentage of how many neighbors got a similar RREQ. Through extensive simulation, it is basically observed that the percentage which will improve a network performance is $75 \%$.Hence, it is clear that when more than $75 \%$ of $\mathrm{CN}$ neighbors obtained a similar RREQ, the CN must not rebroadcast such an obtained one as most of its neighbors got it, so it will not be necessary to rebroadcast it. When lower than $75 \%$ of $\mathrm{CN}$ neighbors get a similar RREQ, it will be rebroadcasted by the CN. Fig. 6 illustrates the overhead / network link in case that CNs have a predefined percentage of what concerns the rebroadcasting of received RREQs.

As a matter of fact, the results presented in Fig. 6 demonstrate what follows: If the percentage is low, overhead will be as well low and vice versa. In other words, if fewer $\mathrm{CN}$ neighbors obtain a similar RREQ, the CN node will rebroadcast that obtained RREQ, thus the addition of more overhead to the network. Actually, in case this percentage is low, most CNs may keep such a RREQ. As a consequence, to find the intended destination will be improbable since few nodes will get the RREQ. From that reason, the balance between reachability and overhead added in the network is struck through the means of setting the percentage at $75 \%$.

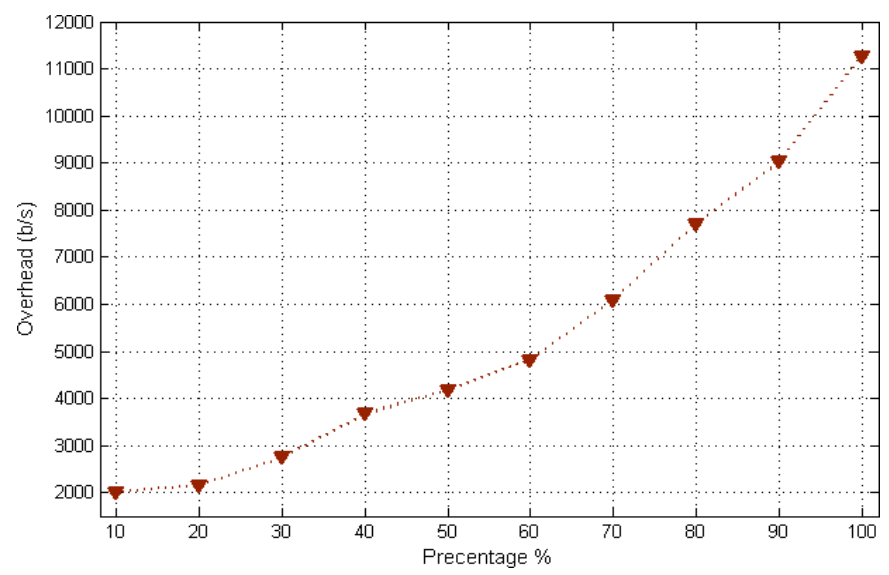

Fig. 6. CN Rebroadcasting Effect. 


\section{PSN PERFORMANCE EVALUATION AND RESULTS ANALYSIS}

The PSN protocol was implemented in the NS2 modeler [23], version 2.34. NS2 is a discrete event network simulator tool used to a great extent when simulating real network scenarios. Added to that, it is freely available and was in the first place designed to simulate wired networks. On the other hand, it has been extended for the simulation of wireless networks including MANETs, wireless LANs and wireless sensor networks. Moreover, it can be organized as it is stated by the OSI reference model [24]. It was shown in [25] that $57 \%$ of all published papers based on simulation- utilized NS2 as their simulation tool. This confirms and demonstrates that NS2 is a network simulator which is powerful and trusted.

\section{A. Simulation Environment and Parameters}

The suggested PSN protocol is exhaustively analyzed through its comparison with our previous proposed A-LSEA and C-CNRR schemes, while depicting its performance. The following section will discuss in detail the results got after comparing between AODV, C-CNRR, A-LSEA and PSN through the use of the parameters given in Table 2. For the simulation of mobile nodes random way points are utilized, where every node will randomly move at a consistent $[5-30$ $\mathrm{m} / \mathrm{s}$ ] speed. At the same time that any node attains one definite random destination, it will take a pause of only two seconds. Afterwards, it will start moving again to a new random destination.

TABLE II. SimUlation PARAMETERS FOR COMPARING AODV, CCNRR, A-LSEA AND PSN

\begin{tabular}{|l|l|}
\hline Simulation area & $600 \times 600 \mathrm{M} 2$ \\
\hline Nodes number & 100 \\
\hline Data rate & $2 \mathrm{Mbps}$ \\
\hline Transmission range & $250 \mathrm{~m}$ \\
\hline Mac protocol & 802.11 \\
\hline Traffic type & CBR \\
\hline Packet size & 1000 bits \\
\hline Traffic & 5 packets $/ \mathrm{sec}$ \\
\hline Simulation time & $600 \mathrm{sec}$ \\
\hline Speed & {$[5 \mathrm{~m} / \mathrm{s}-30 \mathrm{~m} / \mathrm{s}]$} \\
\hline
\end{tabular}

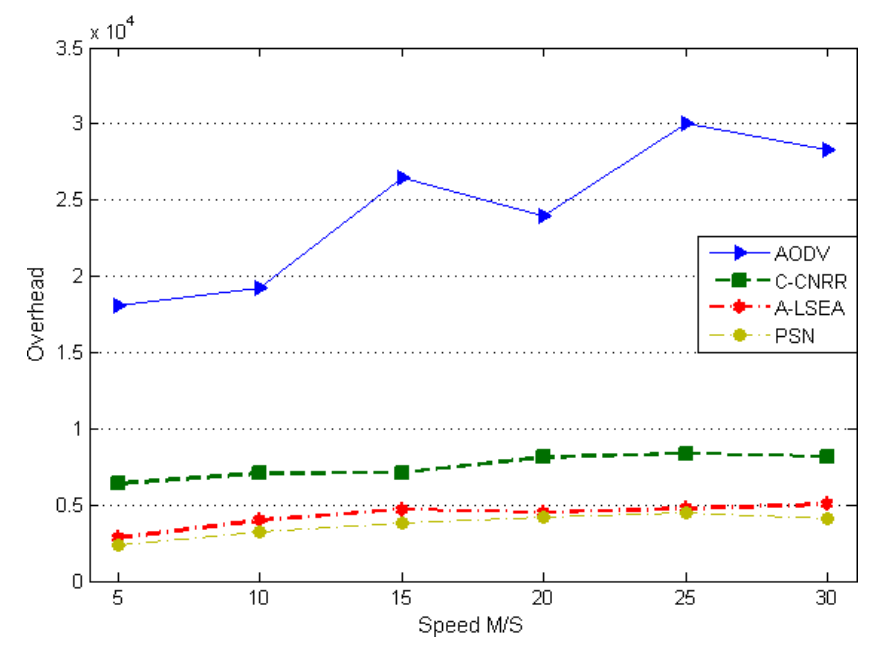

Fig. 7. Overhead Vs. Speed.

\section{B. Results and Discussion for the First Simulation}

This sub-section will analyze in detail the obtained results and it will present the comparative discussion.

1) Total overhead: Fig. 7 shows our comparison of all the overhead of proposed schemes to the AODV, C-CNRR and ALSEA overheads. Fig. 7 demonstrates also that the overhead goes up significantly when mobility grows for AODV. By way of contrast, this rise is constant for these suggested PSN, A-LSEA and C-CNRR schemes. This due to the fact that the AODV protocol will flood any obtained RREQ with no constraints That is to say, without any energy level or link quality. Through the comparison of the other three schemes, it is clear that PSN outperforms A-LSEA and C-CNRR, as long as the PSN will consider LLTAVG and REAVG and will select as well a specific set of nodes (CNs) in the aim of rebroadcasting a RREQ. In addition to that, the PSN routing protocol will reduce to the least possible the overhead via the driving of the CNs to verify the exact number of their own neighbors obtaining a similar RREQ before sending it. On the other hand, C-CNRR considers only the distance,. In spite of that, A-LSEA considers both constraints. This is actually done without a zoning concept or even an extra verification of the number of neighbors receiving the same RREQ.

2) Sent and received RREQs: In the entire network, the number of sent and received RREQs is illustrated in Fig .8. Generally, a broadcast RREQ is sent by one node and afterwards all its neighbors receive it. As a matter of fact, there is a correlation between the number of sent RREQs and the number of received ones (high - high or low -low). The PSN outperforms all other protocols due to the fact that the suggested algorithm selects CNs on the basis of link quality and energy levels as well as the basis of how many node neighbors obtaining one RREQ. When a definite or specific number of ' $S$ ' node neighbors receive one RREQ, the latter will not be flooded within the network. Consequently, there will be across the entire network more control over RREQ dissemination. In the same way, A-LSEA performs better than C-CNRR due to the fact that A-LSEA path selection is more constant (in case that RE and LLT are considered) compared with C-CNRR (considering just the distances between nodes).

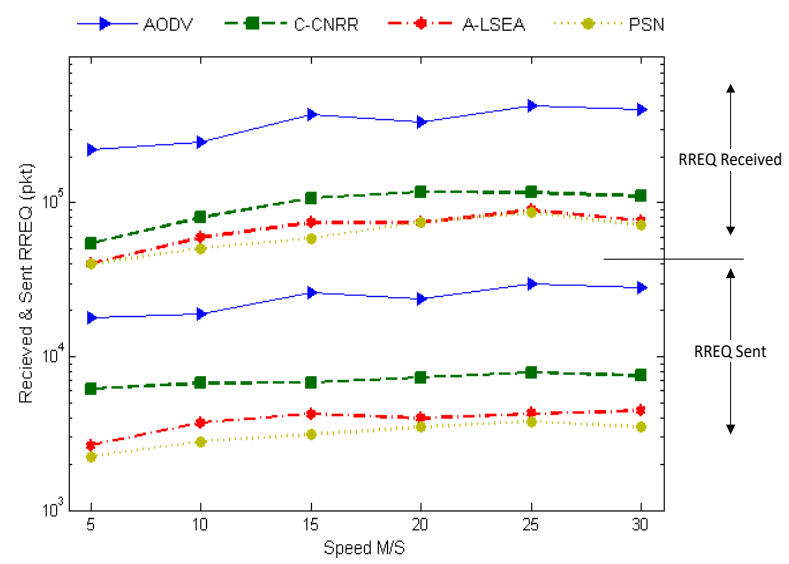

Fig. 8. Received and Sent RREQs Vs. Speed. 


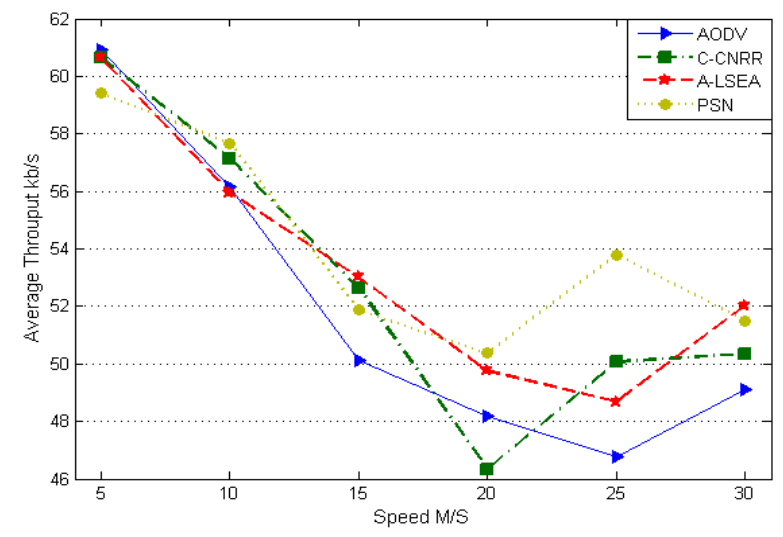

Fig. 9. Throughput Vs. Speed.

3) Average throughput: We demonstrate in Fig. 9 the average PSN routing protocol throughput while comparing it with other routing protocols (A-LSEA, C-CNRR and AODV).

In general, we can see that it decreases when nodes mobility increases for all analyzed protocols. In addition to that, the PSN has a good performance compared to other protocols as the PSN-selected paths holds out more time than the ones selected by other protocols. As a result, the PSN is better than the other protocols (A-LSEA, C-CNRR and AODV) since having the ability to send more data because of very good path lifetimes.

4) Data received: We show in Fig. 10 the data received for PSN as well as the other routing protocols (A-LSEA, CCNRR and AODV)., for which it is demonstrated that the amount of received data will decrease in case mobility increases. This has an effect on the established routes and links. These latter require being re-established whenever breakages occur. By way of contrast, the amount of received data in the PSN routing protocol will decrease in case the speed rises from $5 \mathrm{~m} / \mathrm{s}$ to $15 \mathrm{~m} / \mathrm{s}$. On the other hand, it stays approximately constant above $15 \mathrm{~m} / \mathrm{s}$ for the reason that the PSN-algorithm links judge residual energy and link lifetimes. This makes easier to have an impact on high speeds through the involvement of just the nodes selected by the developed algorithm (the selection of a one best node in every zone). This is performed in an end-to-end path.

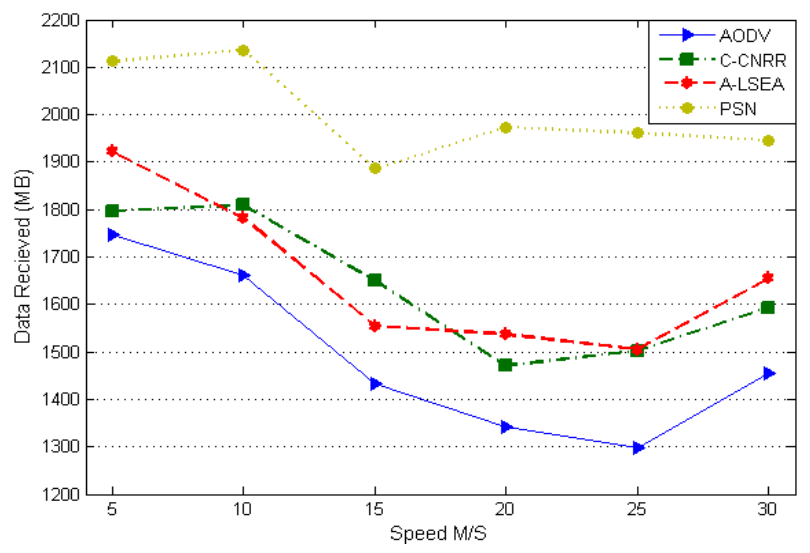

Fig. 10. Data Received vs. Speed.

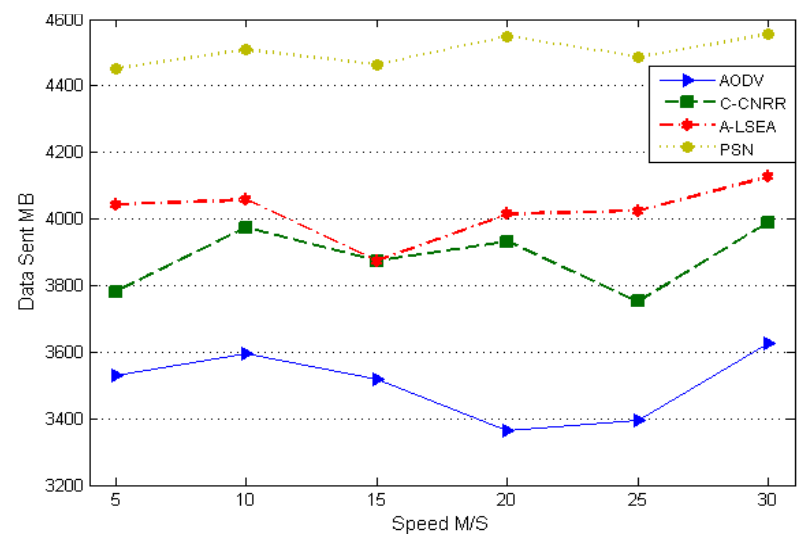

Fig. 11. Data Sent Vs. Speed.

5) Data sent: Fig. 11 depicts the amount of information that has been sent during simulation in a successful manner. The node power supply in MANETs is not permanent because it is naturally mobile. Therefore, any sent or obtained information to and by a node will lead to the reduction in energy levels. In Fig. 8, it can be noticed that AODV is the worst protocol as regards sent or obtained RREQs. A big number of sent or obtained RREQs that are not necessary will greatly decrease the battery life of nodes. Added to that, the PSN is a better protocol when compared to any other routing protocols since it sends a lower number of RREQs, even though it sends more data successfully.

6) Network lifetime: We illustrate in Fig. 12 the proposedPSN, A-LSEA, C-CNRR and AODV network lifetime results. . We show as well that all other routing protocols are outperformed by the PSN, while giving better Network Lifetime results, due to the fact that the PSN routing protocol will select just 4 nodes for rebroadcasting received RREQs.

In addition to that, an advanced algorithm is run by the selected $\mathrm{CN}$ nodes in the goal of eliminating RREQ redundancy by verifying the exact number of their neighbors receiving the same one. According to this, the $\mathrm{CN}$ nodes will discard or rebroadcast the obtained RREQs. AS a matter of fact, saving energy will lead to node energy, hence the growth in network lifetime.

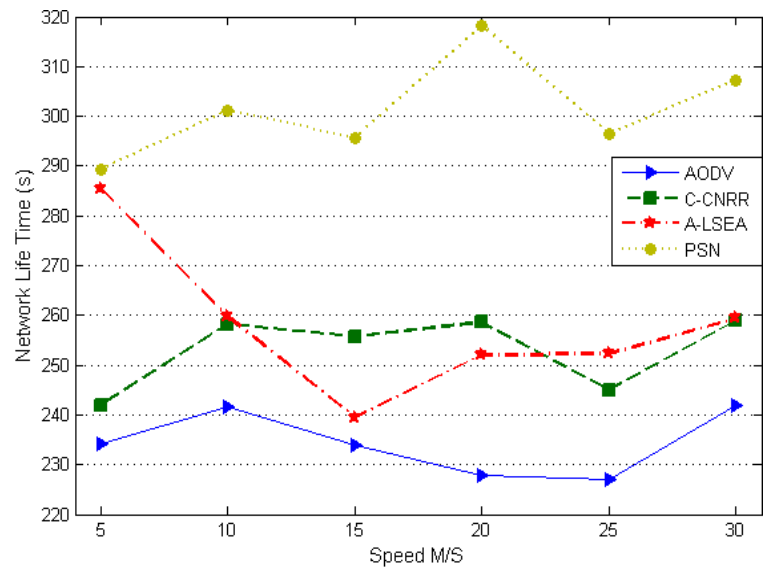

Fig. 12. Network Life Time vs. Speed. 


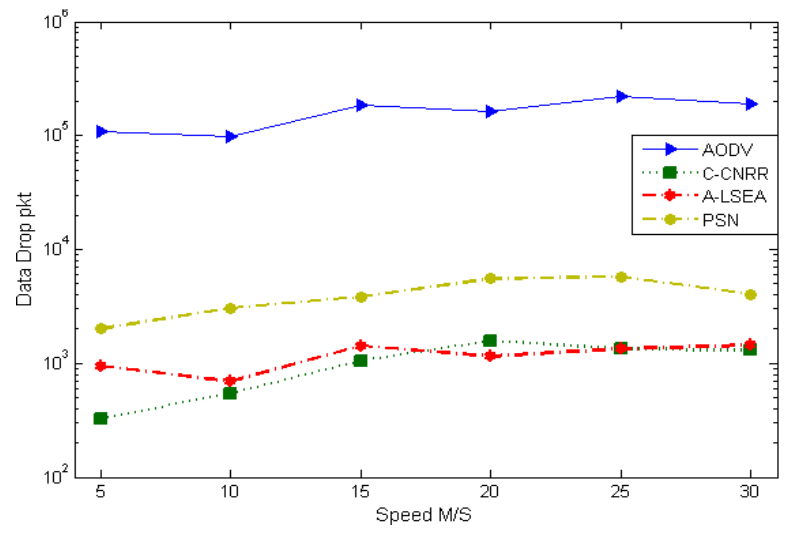

Fig. 13. Data Drop vs. Speed.

7) Data drop: We depict in Fig. 13 (in packets) the amount of data dropping. This is carried out during simulation of proposed-PSN, A-LSEA, C-CNRR and AODV protocols. It is clear that the PSN routing protocol is better than the other routing protocols for these former performance metrics.

On the other hand, Fig. 13 shows that C-CNRR is greatly better than all the other routing protocols in relation to the dropping of data. Despite the fact that the PSN selects better paths compared in fact to the other routing protocols, there will be no advantage of performing better basically as regards any dropped data packets within the network. This can be because the end-to-end-path C-CNRR selection is made on the basis that the distance between the route nodes is advantageous owing to the signal strength for sending and receiving data in distances smaller than those of the routing protocols.

\section{Results and Discussion for Second Simulation}

For further verification and validation, the authors in [20] implemented Mobility-Aware AODV in NS2. It was also compared with the proposed PSN approach. The latter is used in this section in the goal of achieving a better performance while taking into account our previously introduced (A-LSEA and C-CNRR) routing protocols as well as that standard AODV. For this reason, we consider this approach (PSN) in this paper the best proposed routing protocol. In fact, PSN is selected to be compared to AODV and the work suggested in [20] through the use of similar simulation parameters, as provided by Table 3 .

TABLE III. SIMULATION PARAMETERS FOR COMPARING AODV, MAAODV AND PSN

\begin{tabular}{|l|l|}
\hline Simulation area & $700 \times 700 \mathrm{M} 2$ \\
\hline Nodes number & 100 \\
\hline Data rate & $2 \mathrm{Mbps}$ \\
\hline Transmission range & $250 \mathrm{~m}$ \\
\hline Mac protocol & 802.11 \\
\hline Traffic type & CBR \\
\hline Trafic number & 15 flows \\
\hline Packet size & 1000 bits \\
\hline Traffic & 5 packets/sec \\
\hline Simulation time & $700 \mathrm{sec}$ \\
\hline Pause time & {$[2 \mathrm{~s}-12 \mathrm{~s}]$} \\
\hline
\end{tabular}

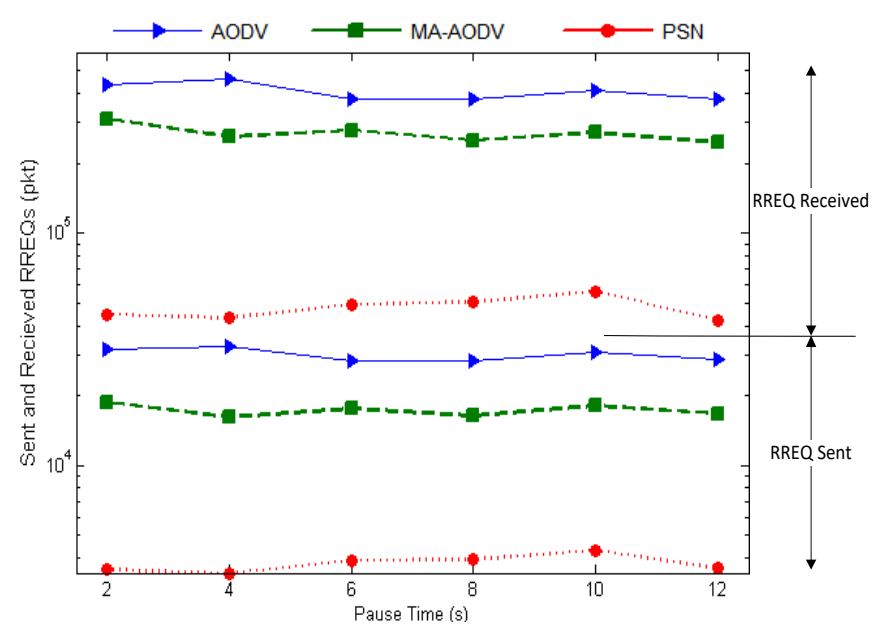

Fig. 14. Sent and Received RREQs vs. Pause Time.

In the next through Fig. 14 - Fig. 20, we will illustrate different metrics [30-32] for the comparisons between PSN, MA-AODV and AODV. It is clear in Fig. 14 that the PSN routing protocol was able to send and receive in the network fewer RREQ packets. This is due to the fact that end-to-end routes are selected by the PSN on the basis of LLT and RE factors

On the other hand routes are selected by MA-AODV only on the basis of node speeds. An edge is given to the PSN over MA-AODV by these two factors for the reason that the route selected by PSN endures for more time than that selected by MA-AODV.

Added to that, those routes selected by MA-AODV endure generally less. Afterwards, the nodes will establish a novel path through the initiation of a new RREQ discovery process. As a consequence, many RREQs will be sent and received in addition to the entire overhead, as depicted in Fig. 15.

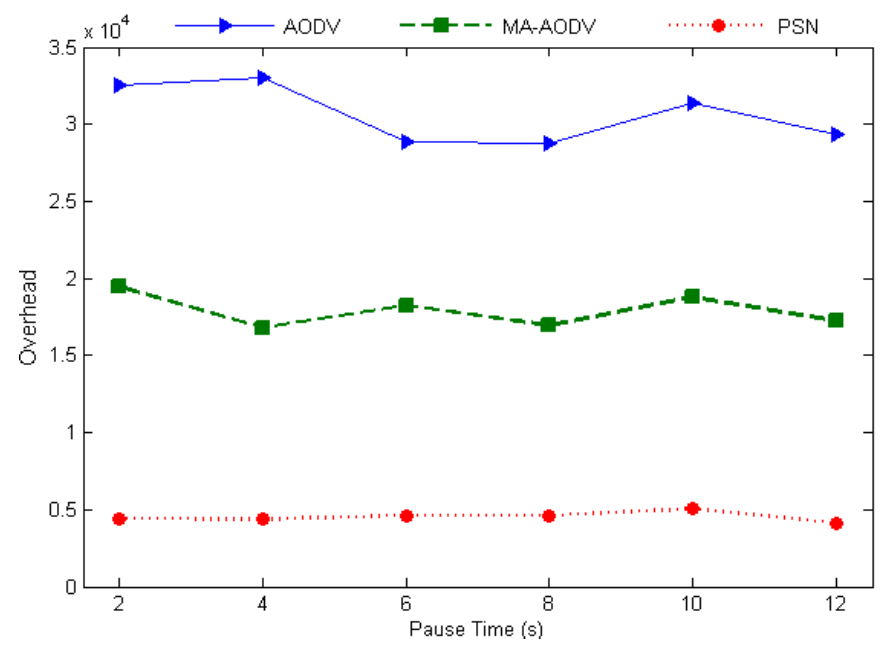

Fig. 15. Average Overhead vs. Pause Time.

The average delivery ratio is illustrated in Fig. 16 for the AODV, MA-AODV and PSN routing protocols. We can see that all routing protocol delivery ratios go up as soon as the pause time increases. 


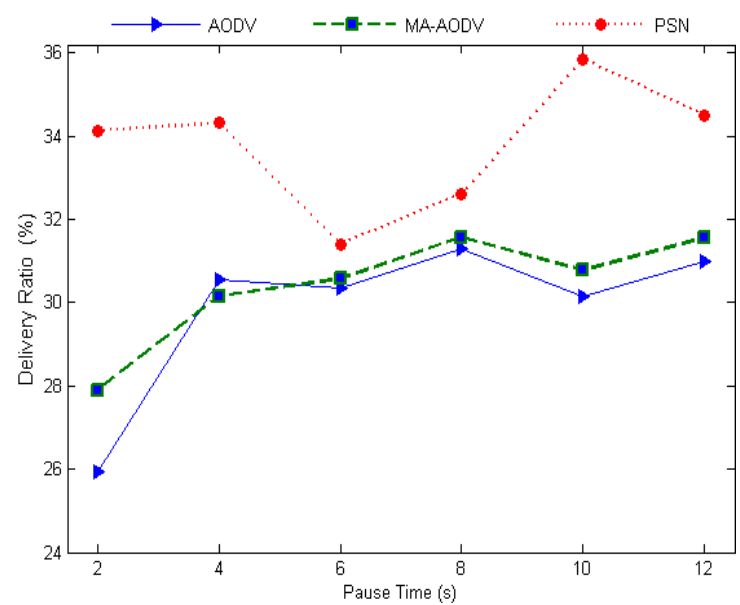

Fig. 16. Delivery Ratio vs. Pause Time.

This is because of the stillness in the mobile nodes. What is more, this PSN routing protocol performs in a good way compared to MA-AODV and AODV routing protocols due to the fact that PSN selects paths lasting longer compared to the ones selected by MA-AODV for the reason that selected PSN end-to-end paths are based on Link Lifeitmes (LLT) and Residual Energy (RE) of the nodes involved in the route. By way of contrast, MA-AODV forwards node-speed-based RREQs. As a result, this MA-AODV algorithm will lead to the end-to-end routes which are entirely having along the path low-speed nodes. On the other hand, this approach, does not ensure any very good paths as regards MANETs because, first of all, , there may be two slow oppositely moving nodes.

As a matter of fact, these two nodes link lifetime can terminate since they move apart. In addition to that, imagine two neighboring nodes that move quite quickly in the same direction. By way of contrast, these two nodes link lifetime is valid for a long time compared to two oppositely moving lowspeed nodes. MA-AODV will just consider that node speed in order to forward a received RREQ. At the same time, the PSN will consider both direction and speed and will calculate in fact the any two neighbor nodes link lifetimes. In the second place, the PSN will provide a very strong packet delivery ratio. This is on account of how the nodes Residual Energies are considered by the PSN during the route selection decision.

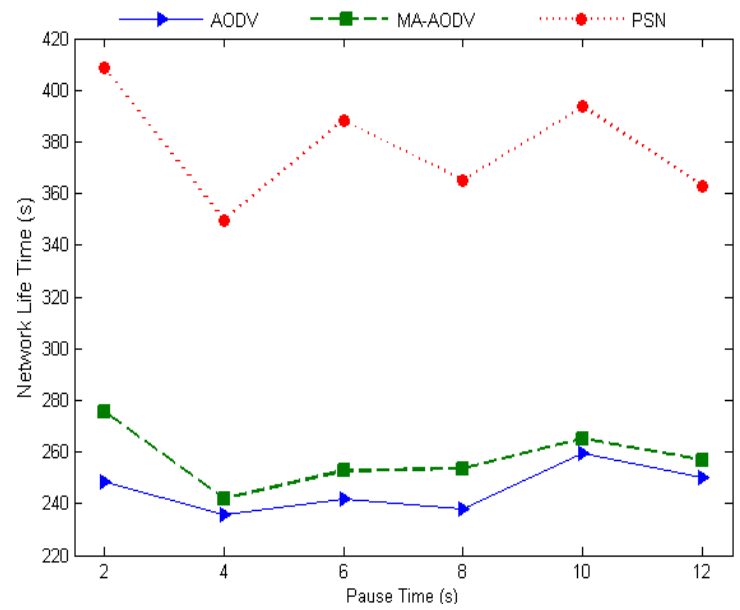

Fig. 17. Network Life Time vs. Pause Time.
Moreover, it is noticeable in Fig. 17 that this suggested PSN runs in a successful way the network for more time compared to the MA-AODV routing protocol. In the first place, the PSN considers the Link Lifetimes and the Residual Energies of the nodes that are in fact involved within end-toend routes whose role is returning stable paths.

Secondly, energy is conserved by discontinuing sending/receiving RREQ packets which are not necessary and which consume a big amount of the node energy. In the same vein, MA-AODV considers just the nodes speed is the latter cannot be an accurate parameter for the selection of unchanging paths. Running a network much longer will enable nodes to send and receive data lot of information, as depicted in Fig. 18. As a result, the PSN outperforms MA-AODV as it sends/ receives more data packets.

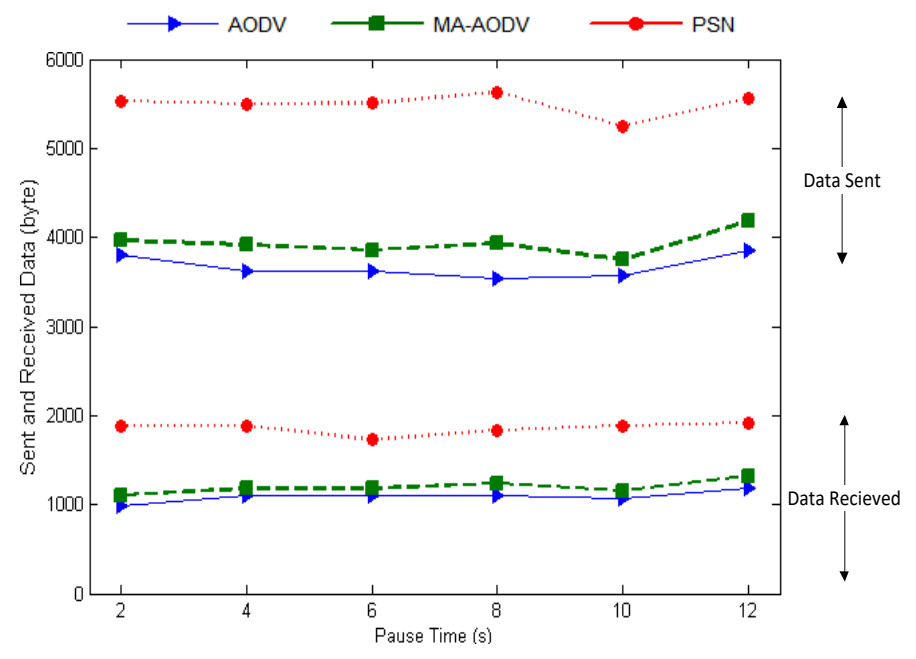

Fig. 18. Sent and Received RREQs vs. Pause Time.

A throughput comparison of AODV, MA-AODV and PSN routing protocols is clearly shown in Fig. 19, where the PSN mostly outperforms MA-AODV on account of that improved algorithm, which improves and stabilizes in a better way endto-end paths.

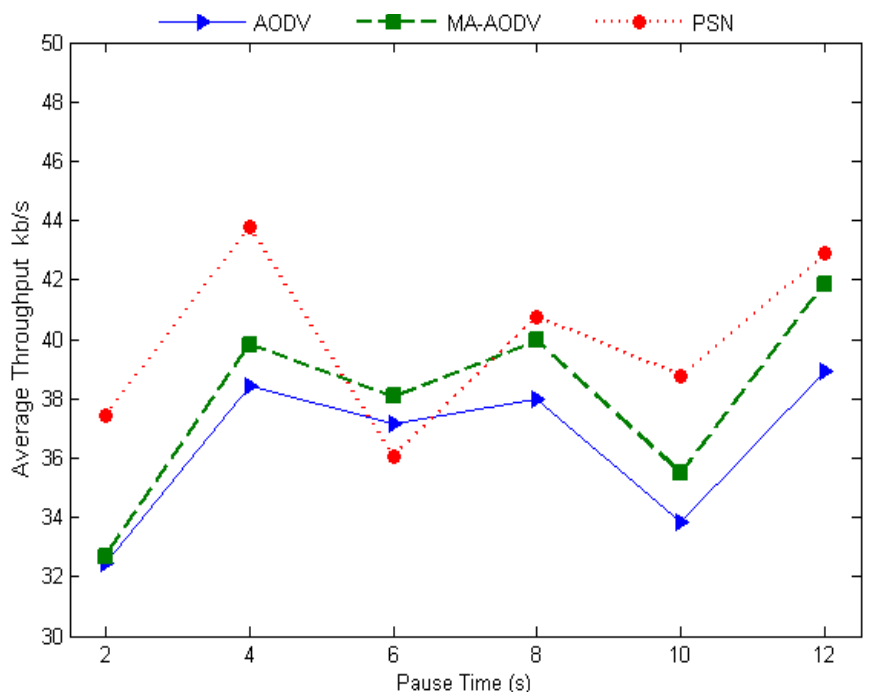

Fig. 19. Average Throughput vs. Pause Time. 


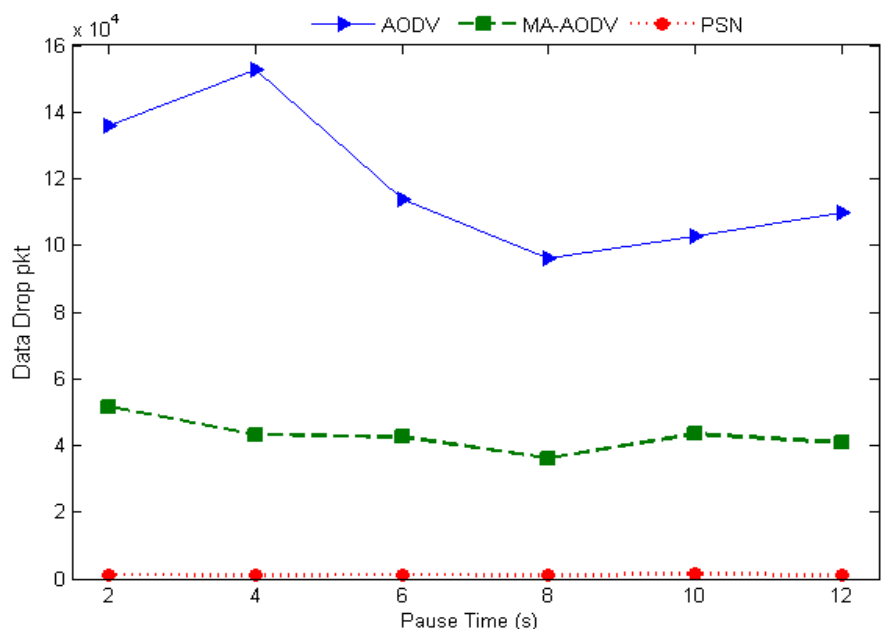

Fig. 20. Data Drop vs. Pause Time.

We can notice as well that the shape of the general throughput rate curve is incremental to the same degree as the pause times increases. This is in spite of some fluctuations caused by nodes mobility randomness. Theoretically speaking, the sex-second-pause-time scenario throughput rate should be greater than a scenario that has a four-second pause time, while having during simulation identical trajectories travelled by nodes. On the other hand, the positions towards which nodes move, under Random Waypoint mobility models, are chosen in a random way and vary from a scenario to another.

Finally, we note that the PSN outperforms the MA-AODV routing protocol as regards the information drop packets, as depicted in Fig. 20, That can be owing to the abovementioned reasons.

\section{CONCLUSION}

This paper presents the PSN protocol, a routing protocol for controlling RREQ propagation within networks, which allows end-to-end paths to be selected based on the Residual Energy (RE) and the Link Lifetimes (LLT). PSN benefits from the combination of these two important factors. Moreover, when the CNRR [27] and LSEA [28. 29] concepts are merged, the RREQs dissemination into the network will actually be reduced without causing reachability loss between the nodes. In addition, we introduced a threshold percentage based method, in which the nodes verify that their neighbors have received before rebroadcasting a RREQ. By preventing nodes from sending duplicate RREQs, this mechanism more intelligently controls network-wide flooding, based on a defined threshold relating to the percentage of its neighbors that have received the RREQ. We performed a simulationbased comparison between the proposed PSN and other routing protocols for different metrics and we have discussed the results.

This increases network lifetimes, improves throughput, and enables more data to be sent and received. The proposed scheme combines both the Residual Energy (RE) and Link Lifetime (LLT) factors in the routing management process, rather than using only a single factor, as in the case studies of $[20,26]$.

\section{ACKNOWLEDGMENT}

Financial support for this study was provided by deanship of Scientific Research, Northern Border University under grant no. (7330-SCI-2017-1-8-F).

\section{REFERENCES}

[1] Perkins, C.E. and Bhagwat, P. "Highly Dynamic Destination-Sequenced distance-vector routing (DSDV) for mobile computers", ACMSIGCOMM Computer Communication Review, vol. 24, no. 4, pp. 234-244, 1994.

[2] Qayyum, A., Jacquet, P. and Muhlethaler, P. "Optimized Link State Routing Protocol (OLSR)", 2003.

[3] Ogier, R., Templin, F. and Lewis, M. (2004) Topology dissemination based on reverse-path forwarding (TBRPF), 2004.

[4] Das, S., Perkins, C. and Royer, E. "Ad hoc on demand distance vector (AODV) routing", Mobile Ad-hoc Network (MANET) Working Group, IETF, 2002 .

[5] Johnson, D.B., Maltz, D.A. and Broch, J. "DSR: The dynamic source routing protocol for multi-hop wireless ad hoc networks", Ad hoc networking, vol. 5, pp. 139-172, 2001.

[6] Z. J. Haas, M. R. Pearlman, and P. Samar, "The Zone Routing Protocol (ZRP) for ad hoc networks," IETF Internet Draft, draft-ietf-manetzonezrp-04.txt., July 2002.

[7] Tseng, Y.C., Ni, S.Y., Chen, Y.S. and Sheu, J.P. "The broadcast storm problem in a mobile ad hoc network", Wireless networks, vol. 8 , no. 2 , pp. 153-167, 2002.

[8] Abdulai, J., Ould-Khaoua, M. and Mackenzie, L. "Improving probabilistic route discovery in mobile ad hoc networks", Local Computer Networks, 2007.LCN 2007. 32nd IEEE Conference, pp. 739, 2007.

[9] Y.-C. Tseng, S.-Y.Ni, and E.-Y. Shih, "Adaptive approaches to relieving broadcast storms in a wireless multihop mobile ad hoc networks," Proceedings of IEEE Transactions on Computers, vol. 52, pp. 545--557, May 2003.

[10] B. Williams and T. Camp, "Comparison of broadcasting techniques for mobile ad hoc networks," Proceedings of the 3rd ACM international symposium on Mobile ad hoc networking \& computing, MOBIHOC, pp. 194 - 205, June 2002.

[11] Le, T.D. and Choo, H. "Efficient flooding scheme based on 2-hop backward information in ad hoc networks", IEEE ICC'08 International Conference on Communications, pp. 2443, 2008.

[12] Yang, S.R., Chiu, C.W. and Yen, W.T. "A novel convex hull-based flooding scheme using 1-hop neighbour information for mobile ad hoc networks", Wireless Networks, vol. 17, no. 7, pp. 1715-1729, 2011.

[13] Liu, H., Wan, P., Jia, X., Liu, X. and Yao, F. "Efficient flooding scheme based on 1-hop information in mobile ad hoc networks", Proc. IEEE INFOCOM, 2006.

[14] Lee, S.H. and Ko, Y.B. "An Efficient Neighbor Knowledge Based Broadcasting for Mobile Ad Hoc Networks", Computational ScienceICCS, pp. 1097-1100, 2006.

[15] Abolhasan, M. and Wysocki, T. "GPS-based route discovery algorithms for on-demand routing protocols in MANETs", Wireless On-Demand Network Systems, pp. 144-157, 2004.

[16] Hamad, S., Noureddine, H., Radhi, N., Shah, I. and Al-Raweshidy, H. "Efficient flooding based on node position for mobile ad hoc network", IEEE Innovations in Information Technology (IIT), pp. 162, 2011.

[17] Yassein, M.B., Khaoua, M.O., Mackenzie, L. and Papanastasiou, S. "Improving the performance of probabilistic flooding in manets", In Proc. of International Workshop on Wireless Ad-hoc Networks (IWWAN), 2005.

[18] Nourazar, F. and Sabaei, M. "DAPF: An Efficient Flooding Algorithm for Mobile Ad-hoc Networks", 2009 International Conference on Signal Processing Systems IEEE, pp. 594, 2009.

[19] Kim J, Zhang Q, Agrawal DP. In: Probabilistic broadcasting based on coverage area and neighbor confirmation in mobile ad hoc networks. Global telecommunications conference workshops, GlobeCom workshops, p. 96-101, 2004. 
[20] Khamayseh Y, Darwish OM, Wedian SA. "Ma-aodv: Mobility aware routing protocols for mobile ad hoc networks", IEEE ICSNC'09 fourth international conference on Systems and networks communications, $\mathrm{p}$. 25-29, 2009.

[21] Hamad S, Noureddine H, Al-Raweshidy H. In: Link stability and energy aware for reactive routing protocol in mobile ad hoc network. Proceedings of the 9th ACM international symposium on mobility management and wireless access; ACM; p. 195-8, 2011.

[22] Kaplan, E.D. and Hegarty, C.J. Understanding GPS: principles and applications, Artech House PublishersK, ISBN 1-58053-894-0, 2006.

[23] "The Network Simulator Ns-2-the VINT pro,"http://www.isi.edu/nsnam/ns/ns-build.html; Retrieved in July 2012.

[24] Lewan, D. and Long, H. "The OSI file service", Proceedings of the IEEE, vol. 71, no. 12, pp. 1414-1419, 1983.

[25] Gast, M. 802.11 wireless networks: the definitive guide, O'Reilly Media, ISBN 0-596-10052-3, 2005.

[26] Xie, F., Du, L., Bai, Y. and Chen, L. "Energy aware reliable routing protocol for mobile ad hoc networks", IEEE Wireless Communications and Networking Conference WCNC, pp. 4313, 2007.
[27] Hamad, Sofian, Salem Belhaj, and Muhana M. Muslam. "Smart Selection of Candidate Neighbors for Efficient Route Discovery in MANETs." Journal of Applied Sciences 17.3 (2017): 126-134.

[28] Hamad, Sofian, Hadi Noureddine, and Hamed Al-Raweshidy. "LSEA: Link stability and energy aware for efficient routing in mobile ad hoc network." Wireless Personal Multimedia Communications (WPMC), 2011 14th International Symposium on. IEEE, 2011.

[29] Hamad, Sofian, Salem Belhaj, and Muhana M. Muslam. "Average Link Stability with Energy-Aware Routing Protocol for MANETs." INTERNATIONAL JOURNAL OF ADVANCED COMPUTER SCIENCE AND APPLICATIONS 9, no. 1 (2018): 554-562.

[30] T. Yeferny, K. Arour, A. Bouzeghoub. "An efficient peer-to-peer semantic overlay network for learning query routing". 27th IEEE International Conference on Advanced Information Networking and Applications (AINA), pp. 1025-1032 (2013).

[31] T. Yeferny, K. Arour. "Efficient routing method in p2p systems based upon training knowledge". 26th IEEE International Conference on Advanced Information Networking and Applications Workshops (WAINA), pp. 300-305 (2012).

[32] Taoufik Yeferny, Sofian Hamad and Salem Belhaj. "CDP: a Content Discovery Protocol for Mobile P2P Systems". International Journal of Computer Science and Network Security, VOL.18 No.5, May 2018 